\title{
Effect of rotation, magnetic field and a periodic loading on radial vibrations thermo-viscoelastic non-homogeneous media
}

\author{
Khalil S Al-Basyouni ${ }^{1 *}$, Samy R Mahmoud ${ }^{1,2}$ and Ebraheem O Alzahrani ${ }^{1}$
}

\author{
"Correspondence: \\ kalbasyouni@kau.edu.sa \\ ${ }^{1}$ Mathematics Department, Faculty \\ of Science, King Abdulaziz \\ University, P.O. Box 80203, Jeddah, \\ 21589, Saudi Arabia \\ Full list of author information is \\ available at the end of the article
}

\begin{abstract}
In this paper, an analytical solution for the effect of the rotation in a magnetothermo-viscoelastic non-homogeneous medium with a spherical cavity subjected to periodic loading is presented. The distribution of displacements, temperature, and stresses in the non-homogeneous medium in the context of generalized thermo-elasticity using GL (Green-Lindsay) theory is discussed and obtained in analytical form. The results are displayed graphically to illustrate the effect of rotation, relaxation, magnetic field, viscoelasticity, and non-homogeneity. Comparisons are made with the previous work in the absence of rotation and initial stress.
\end{abstract}

Keywords: rotation; viscoelasticity; periodic loading; non-homogeneity; magneto-thermo-elasticity

\section{Introduction}

In recent years, the theory of magneto-thermo-viscoelasticity, which deals the interactions among strain, temperature, and electromagnetic fields has drawn the attention of many researchers because of its extensive use in diverse fields, such as geophysics for understanding the effect of the Earth's magnetic field on seismic waves, damping of acoustic waves in a magnetic field, emission of electromagnetic radiations from nuclear devices, development of a highly sensitive superconducting magnetometer, electrical power engineering, optics, etc.; see [1-4]. Mahmoud et al. [5, 6] investigated the effect of the rotation on plane vibrations in a transversely isotropic infinite hollow cylinder, the effect of the rotation on wave motion through a cylindrical bore in a micropolar porous cubic crystal and he investigated the effect of a magnetic field and non-homogeneity on the radial vibrations in a hollow rotating elastic cylinder. Abd-Alla et al. [7-10] investigated the effect of the rotation on a non-homogeneous infinite cylinder of orthotropic material, influence of rotation, radial vibrations in a non-homogeneous orthotropic elastic hollow sphere subjected to rotation, and they investigated the magneto-thermo-elastic problem in a rotating non-homogeneous orthotropic hollow cylinder in the hyperbolic heat conduction model. Mahmoud $[11,12]$ investigated the analytical solution for an electrostatic potential on wave propagation modeling in human long wet bones, and they studied the influence of rotation and generalized magneto-thermo-elastics on Rayleigh waves in a granular medium under

(c) 2014 Al-Basyouni et al.; licensee Springer. This is an Open Access article distributed under the terms of the Creative Commons Attribution License (http://creativecommons.org/licenses/by/4.0), which permits unrestricted use, distribution, and reproduction in any medium, provided the original work is properly credited. 
the effect of initial stress and a gravity field. Abd-Alla and Mahmoud [13] investigated the analytical solution of wave propagation in non-homogeneous orthotropic rotating elastic media. Abd-Alla et al. [14-16] investigated some problems like the propagation of an $\mathrm{S}$-wave in a non-homogeneous anisotropic incompressible and initially stressed medium under the influence of a gravity field, the generalized magneto-thermo-elastic Rayleigh waves in a granular medium under the influence of a gravity field and initial stress, and they also investigated the problem of transient coupled thermo-elasticity of an annular fin. Some problems of thermo-elasticity and wave propagation modeling in a cylinder are investigated by Abd-Alla et al. [17, 18], respectively. Mukhopadhyay [19] investigated the effects of thermal relaxations on thermo-viscoelastic interactions in an unbounded body with a spherical cavity subjected to a periodic loading on the boundary. The effects of thermal relaxations on thermo-elastic interactions in an unbounded body with a spherical cavity or cylindrical hole subjected to a periodic loading on the boundary, respectively, were investigated by Roychoudhuri and Mukhopadhyay [20]. The thermally induced vibrations in a generalized thermo-elastic solid with a cavity have been investigated by Erbay et al. [21] and Li and Qi [22]. Mahmoud [23] investigated the analytical solution for free vibrations of an elasto-dynamic orthotropic hollow sphere under the influence of rotation.

In this paper, rotation and the magneto-thermo-elastic equation of a spherical cavity are decomposed into a non-homogeneous equation with boundary conditions. The effect of thermal relaxation times on the wave propagation in the magneto-thermo-viscoelastic case using the GL theory will be discussed. We take the material of the spherical cavity to be of Kelvin-Voigt type. Thus, the exact expressions for the transient response of displacement, stresses, and temperature in a spherical cavity are obtained. The numerical calculations will be investigated for the displacement, temperature, and the components of stresses, and we explain the special case from this study when the magnetic field and non-homogeneity are neglected. Finally, numerical results are calculated and discussed.

\section{Formulation of the problem}

We shall consider the spherical coordinates of any representing point to be $(r, \theta, \phi)$ and assume that the spherical cavity is subjected to a rapid change in temperature $T(r, t)$ and magnetic field $\bar{H}\left(0,0, H_{0}\right)$, for the axisymmetric plane strain problem, and the components of the displacement $\bar{u}=\bar{u}\left(u_{r}, u_{\theta}, u_{\phi}\right)$ are expressed as $u_{\theta}=u_{\phi}=0$, and $u_{r}=u_{r}(r, t)$. Let us consider an infinite isotropic non-homogeneous viscoelastic solid, and the viscoelastic nature of the material is described by the Voigt type of linear viscoelasticity. The medium is assumed to have a spherical cavity of radius $a$. For a spherical symmetric system, the non-vanishing stress components are expressed as

$$
\begin{aligned}
& \tau_{r r}=\tau_{m}(\lambda+2 \mu) \frac{\partial u_{r}}{\partial r}+2 \lambda \tau_{m} \frac{u_{r}}{r}-\gamma\left(T+\tau_{2} \dot{T}\right), \\
& \tau_{\theta \theta}=2 \tau_{m}(\lambda+\mu) \frac{u_{r}}{r}+\lambda \tau_{m} \frac{\partial u_{r}}{\partial r}-\gamma\left(T+\tau_{2} \dot{T}\right), \\
& \tau_{\varphi \varphi}=2 \tau_{m}(\lambda+\mu) \frac{u_{r}}{r}+\lambda \tau_{m} \frac{\partial u_{r}}{\partial r}-\gamma\left(T+\tau_{2} \dot{T}\right), \\
& \tau_{r \varphi}=\tau_{r \theta}=\tau_{\theta \varphi}=0,
\end{aligned}
$$

where $\tau_{r r}, \tau_{\theta \theta}$, and $\tau_{\varphi \varphi}$ are the normal mechanical stresses, $\tau_{r \varphi}, \tau_{r \theta}, \tau_{\theta \varphi}$ are shear mechanical stresses. $\tau_{m}=1+\tau_{0} \frac{\partial}{\partial t}$ and $\tau_{0}$ are the mechanical relaxation times due to the viscosity. 
The magneto-elasto-dynamic equation of the non-homogeneity in the spherical case if $u_{r}=u_{r}(r, t)$, becomes

$$
\begin{aligned}
& \frac{\partial \tau_{r r}}{\partial r}+\frac{2}{r} \tau_{r r}-\frac{1}{r} \tau_{\theta \theta}-\frac{1}{r} \tau_{\varphi \varphi}+\mu_{e}(\bar{J} \times \bar{H})-\rho(\overleftarrow{\Omega} \times \overleftarrow{\Omega} \times \overleftarrow{u})_{r}-\rho(2 \overleftarrow{\Omega} \times \overleftarrow{u})_{r} \\
& \quad=\rho \frac{\partial^{2} u_{r}}{\partial t^{2}}
\end{aligned}
$$

where $\overleftarrow{\Omega}=(\Omega, 0,0), \overleftarrow{\Omega} \times \overleftarrow{\Omega} \times \overleftarrow{u}$ is the centripetal acceleration due to the time varying motion only and $2 \overleftarrow{\Omega} \times \overleftarrow{u}$ is the Coriolis acceleration, we have the Lorentz force [18], which may be written as

$$
\begin{aligned}
& f_{r}=\mu_{e}(\bar{J} \times \bar{H})=\mu_{e} H_{0}^{2} \frac{\partial}{\partial r}\left(\frac{\partial u_{r}}{\partial r}+\frac{2 u_{r}}{r}\right), \\
& \bar{h}=\operatorname{curl}(\bar{u} \times \bar{H})=\left(0,0,-H_{0}\left(\frac{\partial u_{r}}{\partial r}+\frac{2 u_{r}}{r}\right)\right), \quad \bar{J}=\operatorname{curl} \bar{h}=\left(0, \frac{\partial h_{\phi}}{\partial r}, 0\right),
\end{aligned}
$$

where $\bar{h}$ is the perturbed magnetic field over the primary magnetic field, $\bar{E}$ is the electric intensity, $\bar{J}$ is the electric current density, $\mu_{e}$ is the magnetic permeability, $\bar{H}$ is the constant primary magnetic field, and $\bar{u}$ is the displacement vector.

The magneto-thermo-elasto-dynamic equations of the non-homogeneity sphere may be written as

$$
\begin{aligned}
& \frac{\partial \tau_{r r}}{\partial r}+\frac{2}{r} \tau_{r r}-\frac{1}{r} \tau_{\theta \theta}-\frac{1}{r} \tau_{\varphi \varphi}+\mu_{e} H_{0}^{2} \frac{\partial}{\partial r}\left(\frac{\partial u_{r}}{\partial r}+\frac{2 u_{r}}{r}\right)+\rho \Omega^{2} u_{r}=\rho \frac{\partial^{2} u_{r}}{\partial t^{2}}, \\
& L\left(\frac{\partial^{2} \theta}{\partial r^{2}}+\frac{2}{r} \frac{\partial \theta}{\partial r}\right)=\rho c_{\nu}\left(\frac{\partial \theta}{\partial t}+\tau_{1} \frac{\partial^{2} \theta}{\partial t^{2}}\right)+\gamma T_{0}\left[\frac{\partial}{\partial r}+\frac{2}{r}\right] \dot{u}_{r},
\end{aligned}
$$

where $L$ is the thermal conductivity, $\gamma=\alpha_{t}(3 \lambda+2 \mu), \Omega$ is the rotation, $\rho$ is the density of the material, $c_{v}$ is the specific heat of the material per unit mass, $\tau_{1}, \tau_{2}$ are thermal relaxation parameters, $\alpha_{t}$ is the coefficient of linear thermal expansion, $\lambda, \mu$ are Lame elastic constants, $T_{1}$ is the absolute temperature, $T_{0}$ is a reference temperature of solid, $\theta$ is temperature difference $\left(T_{1}-T_{0}\right)$, and $\tau_{0}$ is the mechanical relaxation time due to the viscosity. In the above formula, the non-homogeneity of the material is characterized by a special law as follows:

$$
\lambda=\lambda_{0} r^{2 n}, \quad \mu=\mu_{0} r^{2 n}, \quad \rho=\rho_{0} r^{2 n}, \quad \mu_{e}=\mu_{e}^{0} r^{2 n}, \quad \gamma=\gamma_{0} r^{2 n}
$$

where $\lambda_{0}, \mu_{0}, \rho_{0}$ and $\mu_{e}^{0}$ are the Lame's constant, shear modulus, mass density, pressure, and magnetic permeability coefficient of the homogeneous material, respectively, and $n$ expresses a non-homogeneous exponent of the material, which is an arbitrary real number. Substituting (5) into (1) yields

$$
\begin{aligned}
\tau_{r r} & =r^{2 n}\left[\tau_{m}\left(\lambda_{0}+2 \mu_{0}\right) \frac{\partial u_{r}}{\partial r}+2\left(\lambda_{0}\right) \tau_{m} \frac{u_{r}}{r}-\gamma_{0}\left(\theta+\tau_{2} \dot{\theta}\right)\right], \\
\tau_{\theta \theta} & =r^{2 n}\left[2 \tau_{m}\left(\lambda_{0}+\mu_{0}\right) \frac{u_{r}}{r}+\left(\lambda_{0}\right) \tau_{m} \frac{\partial u_{r}}{\partial r}-\gamma_{0}\left(\theta+\tau_{2} \dot{\theta}\right)\right],
\end{aligned}
$$




$$
\begin{aligned}
\tau_{\varphi \varphi} & =r^{2 n}\left[2 \tau_{m}\left(\lambda_{0}+\mu_{0}\right) \frac{u_{r}}{r}+\lambda_{0} \tau_{m} \frac{\partial u_{r}}{\partial r}-\gamma_{0}\left(\theta+\tau_{2} \dot{\theta}\right)\right], \\
\tau_{r \varphi} & =\tau_{r \theta}=\tau_{\theta \varphi}=0, \\
\sigma_{r r}^{*} & =\mu_{e}^{0} H_{0}^{2} r^{2 n}\left(\frac{\partial u_{r}}{\partial r}+\frac{2 u_{r}}{r}\right),
\end{aligned}
$$

where $\sigma_{r r}^{*}$ is the Maxwell stress tensor. From plugging (5) and (6) into (2) we have

$$
\begin{aligned}
& {\left[\tau_{m}+\frac{\mu_{e}^{0} H_{\phi}^{2}}{\left(\lambda_{0}+2 \mu_{0}\right)}\right] \frac{\partial^{2} u_{r}}{\partial r^{2}}+\left[2(n+1) \tau_{m}+\frac{\mu_{e}^{0} H_{\phi}^{2}}{\left(\lambda_{0}+2 \mu_{0}\right)}\right] \frac{1}{r} \frac{\partial u_{r}}{\partial r}} \\
& +\left[\frac{4 n \lambda_{0} \tau_{m}}{\left(\lambda_{0}+2 \mu_{0}\right)}-2 \tau_{m}-\frac{\mu_{e}^{0} H_{\phi}^{2}}{\left(\lambda_{0}+2 \mu_{0}\right)}\right] \frac{u_{r}}{r^{2}}-\left[\frac{2 n}{r}+\frac{\partial}{\partial r}\right] \frac{\gamma_{0}}{\left(\lambda_{0}+2 \mu_{0}\right)}\left(\theta+\tau_{2} \dot{\theta}\right) \\
& +\rho_{0} r^{2 n} \Omega^{2} u=\frac{\rho_{0}}{\left(\lambda_{0}+2 \mu_{0}\right)} \frac{\partial^{2} u}{\partial t^{2}} .
\end{aligned}
$$

Let $c_{0}=\frac{\lambda_{0}}{\left(\lambda_{0}+2 \mu_{0}\right)}, c_{2}=\frac{\gamma_{0}}{\left(\lambda_{0}+2 \mu_{0}\right)}, c_{3}=\frac{\mu_{e}^{0} H_{\phi}^{2}}{\left(\lambda_{0}+2 \mu_{0}\right)}, c_{\nu}=\sqrt{\frac{\left(\lambda_{0}+2 \mu_{0}\right)}{\rho_{0}}}$.

Then the elasto-dynamic equation (7) becomes

$$
\begin{gathered}
\left(\tau_{m}+c_{3}\right) \frac{\partial^{2} u_{r}}{\partial r^{2}}+\left[2(n+1) \tau_{m}+c_{3}\right] \frac{1}{r} \frac{\partial u_{r}}{\partial r}+\left[4 n c_{0} \tau_{m}-2 \tau_{m}-c_{3}\right] \frac{u_{r}}{r^{2}} \\
-c_{2}\left[\frac{2 n}{r}+\frac{\partial}{\partial r}\right]\left(\theta+\tau_{2} \dot{\theta}\right)+\rho_{0} \Omega^{2} u_{r}=\frac{1}{c_{v}^{2}} \frac{\partial^{2} u_{r}}{\partial t^{2}} .
\end{gathered}
$$

In addition, the heat conduction equation is

$$
L\left(\frac{\partial^{2} \theta}{\partial r^{2}}+\frac{2}{r} \frac{\partial \theta}{\partial r}\right)=\rho_{0} r^{2 n} c_{\nu}\left(\frac{\partial \theta}{\partial t}+\tau_{1} \frac{\partial^{2} \theta}{\partial t^{2}}\right)+\gamma_{0} r^{2 n} T_{0}\left[\frac{\partial}{\partial r}+\frac{2}{r}\right] \dot{u}_{r}
$$

We now use the following dimensionless quantities:

$$
\begin{aligned}
& U=\frac{u_{r}}{a}, \quad l=\frac{L}{\rho_{0} c_{v}}, \quad t^{\prime}=\frac{k c_{v}}{a} t, \quad T=\frac{\theta}{T_{0}}, \quad \tau_{0}^{\prime}=\frac{c_{v}}{a} \tau_{0}, \quad \tau_{1}^{\prime}=\frac{c_{v}}{a} \tau_{1}, \\
& \tau_{2}^{\prime}=\frac{k c_{v}}{a} \tau_{2}, \quad r^{\prime}=\frac{r}{a}, \quad \Omega^{*}=\frac{\Omega}{a}, \\
& \sigma_{r r}=\frac{\tau_{r r}}{\left(\lambda_{0}+2 \mu_{0}\right)}, \quad \sigma_{\theta \theta}=\frac{\tau_{\theta \theta}}{\left(\lambda_{0}+2 \mu_{0}\right)} .
\end{aligned}
$$

In the following discussion the primes are neglected for $r^{\prime}$.

The normal stresses relations can be set right in non-dimensional form as:

$$
\begin{aligned}
& \sigma_{r r}=(a r)^{2 n}\left[\left(1+\tau_{0}^{\prime} \frac{\partial}{\partial t^{\prime}}\right) \frac{\partial U}{\partial r}+2 c_{1}\left(1+\tau_{0}^{\prime} \frac{\partial}{\partial t^{\prime}}\right) \frac{U}{r}-T_{0} c_{2}\left(1+\tau_{2}^{\prime} \frac{\partial}{\partial t^{\prime}}\right) T\right], \\
& \sigma_{\theta \theta}=(a r)^{2 n}\left[2\left(1+\tau_{0}^{\prime} \frac{\partial}{\partial t^{\prime}}\right) \frac{U}{r}+c_{4}\left(1+\tau_{0}^{\prime} \frac{\partial}{\partial t^{\prime}}\right) \frac{\partial U}{\partial r}-T_{0} c_{5}\left(1+\tau_{2}^{\prime} \frac{\partial}{\partial t^{\prime}}\right) T\right], \\
& \sigma_{r r}^{*}=\mu_{e}^{0} H_{\phi}^{2}(a r)^{2 n}\left(\frac{\partial U}{\partial r}+\frac{2 U}{r}\right), \quad f_{r}=\frac{\mu_{e}^{0} H_{\phi}^{2}(a r)^{2 n}}{a}\left(\frac{\partial^{2} U}{\partial r^{2}}+\frac{2}{r} \frac{\partial U}{\partial r}-\frac{2 U}{r^{2}}\right),
\end{aligned}
$$

where $c_{4}=\frac{\lambda_{0}+P_{0}^{*}}{\left(\lambda_{0}+2 \mu_{0}\right)}, c_{5}=\frac{\gamma_{0}}{\left(\lambda_{0}+2 \mu_{0}\right)}$. 
Substituting of (11) into (8) and (9) gives the displacement equation in the non-dimensional form of the non-homogeneous spherical as follows:

$$
\begin{aligned}
& {\left[\left(1+\tau_{0}^{\prime} \frac{\partial}{\partial t^{\prime}}\right)+c_{3}\right] \frac{\partial^{2} U}{\partial r^{2}}+\left[2(n+1)\left(1+\tau_{0}^{\prime} \frac{\partial}{\partial t^{\prime}}\right)+c_{3}\right] \frac{1}{r} \frac{\partial U}{\partial r}} \\
& \quad+\left[\left(4 n c_{1}-2\right)\left(1+\tau_{0}^{\prime} \frac{\partial}{\partial t^{\prime}}\right)-c_{3}\right] \frac{U}{r^{2}} \\
& \quad-c_{2} T_{0}\left[1+\tau_{2}^{\prime} \frac{\partial}{\partial t^{\prime}}\right]\left(\frac{2 l}{r}+\frac{1}{c_{L}^{2}} \frac{\partial}{\partial r}\right) T+\rho_{0} a c_{1}^{2} \Omega^{* 2} U=l^{2} \frac{\partial^{2} U}{\partial t^{\prime 2}} .
\end{aligned}
$$

The heat conduction equation in non-dimensional form is

$$
\left(\frac{\partial^{2} T}{\partial r^{2}}+\frac{2}{r} \frac{\partial T}{\partial r}\right)=l_{1}\left(1+\tau_{1}^{\prime} \frac{\partial}{\partial t^{\prime}}\right) \frac{\partial T}{\partial t^{\prime}}+l_{2}\left[\frac{\partial}{\partial r}+\frac{2}{r}\right] \frac{\partial U}{\partial t^{\prime}},
$$

where $l_{1}=\frac{a c_{\nu}}{l}, l_{2}=\frac{a \gamma_{0}}{\rho_{0}}$.

\section{The problem solution}

We seek the general solution to the basic equation (12) of magneto-thermo-elastic motion as a harmonic vibration in the form

$$
\begin{aligned}
& U\left(r, t^{\prime}\right)=U^{*}(r) e^{i \omega t^{\prime}}, \\
& T\left(r, t^{\prime}\right)=T^{*}(r) e^{i \omega t^{\prime}} .
\end{aligned}
$$

From (14) the equation of motion becomes

$$
\begin{aligned}
{\left[\left(1+i \omega \tau_{0}^{\prime}\right)+c_{3}\right] \frac{d^{2} U^{*}}{d r^{2}}+\left[2(n+1)\left(1+i \omega \tau_{0}^{\prime}\right)+c_{3}\right] \frac{1}{r} \frac{d U^{*}}{d r} } \\
+\left[\left(4 n c_{1}-2\right)\left(1+i \omega \tau_{0}^{\prime}\right)-c_{3}\right] \frac{U^{*}}{r^{2}}+\rho_{0} a c_{1}^{2} \Omega^{* 2} U^{*} \\
=-k^{2} \omega^{2} U^{*}+c_{2} T_{0} \gamma^{\prime}\left(\frac{2 n}{r}+\frac{d}{d r}\right) T^{*}
\end{aligned}
$$

where $\gamma^{\prime}=\left(1+i \tau_{2}^{\prime} \omega\right)$, or in the form

$$
\frac{d^{2} U^{*}}{d r^{2}}+\eta_{1} \frac{1}{r} \frac{d U^{*}}{d r}+\eta_{2} \frac{U^{*}}{r^{2}}+\rho_{0} a c_{1}^{2} \Omega^{* 2} U^{*}=-m_{1}^{2} U^{*}+\varepsilon\left(\frac{2 n}{r}+\frac{d}{d r}\right) T^{*},
$$

where

$$
\begin{aligned}
& \eta_{1}=\frac{(2 n+1)\left(1+i \omega \tau_{0}^{\prime}\right)}{\left(1+i \omega \tau_{0}^{\prime}\right)+c_{3}}+1, \quad \eta_{2}=\frac{\left(4 n c_{1}-1\right)\left(1+i \omega \tau_{0}^{\prime}\right)}{\left(1+i \omega \tau_{0}^{\prime}\right)+c_{3}}-1, \\
& \varepsilon=\frac{c_{2} T_{0} \gamma^{\prime}}{\left(1+i \omega \tau_{0}^{\prime}\right)+c_{3}}, \quad m_{1}^{2}=\frac{k^{2} \omega^{2}}{\left(1+i \omega \tau_{0}^{\prime}\right)+c_{3}}, \quad c_{1}=\sqrt{\frac{\lambda+2 \mu}{\rho_{0}}} .
\end{aligned}
$$

Also the heat conduction equation becomes

$$
\left(\nabla^{2}+\beta_{1}\right) T^{*}=\beta_{2}\left[\frac{d}{d r}+\frac{2}{r}\right] U^{*}
$$

where $\nabla^{2}=\frac{d^{2}}{d r^{2}}+\frac{2}{r} \frac{d}{d r}, \beta_{1}=l_{1}\left(\omega^{2} \tau_{1}^{\prime}-i \omega\right), \beta_{2}=i \omega l_{2}$. 
To solve (16) and (17), we let

$$
\begin{aligned}
& U^{*}(r)=\frac{d \xi(r)}{d r}, \\
& \frac{d}{d r}\left[\frac{d^{2} \xi(r)}{d r^{2}}+\frac{\eta_{1}}{r} \frac{d \xi(r)}{d r}+\frac{\eta_{2}}{r^{2}} \xi(r)\right]+f_{1} \Omega^{* 2} \frac{d \xi(r)}{d r}=-m_{1}^{2} \frac{d \xi(r)}{d r}+\varepsilon\left(\frac{2 n}{r}+\frac{d}{d r}\right) T^{*}
\end{aligned}
$$

By comparing the coefficients of $\frac{d}{d r}$ in (19), we get

$$
\frac{d^{2} \xi(r)}{d r^{2}}+\frac{\eta_{1}}{r} \frac{d \xi(r)}{d r}+\left[\frac{\eta_{2}}{r^{2}}+m_{1}^{2}\right] \xi(r)=\varepsilon T^{*}
$$

The heat conduction equation becomes

$$
\left(\nabla^{2}+\beta_{1}\right) T^{*}=\beta_{2} \nabla^{2} \xi(r) .
$$

From (20) and (21), we have

$$
\begin{aligned}
& \frac{d^{4} \xi(r)}{d r^{4}}+\left[\eta_{1}+1\right] \frac{1}{r} \frac{d^{3} \xi(r)}{d r^{3}}+\left[\Gamma_{1}+\frac{\eta_{2}-\eta_{1}}{r^{2}}\right] \frac{d^{2} \xi(r)}{d r^{2}} \\
& \quad+\left[\frac{\eta_{2}+\eta_{1}}{r^{3}}+\frac{\Gamma_{2}}{r}\right] \frac{d \xi(r)}{d r}+\beta_{1} N^{2} \xi+f_{1} \Omega^{* 2} \frac{d \xi(r)}{d r}=0,
\end{aligned}
$$

where $\Gamma_{1}=m_{1}^{2}+\beta_{1}-\varepsilon \beta_{2}, \Gamma_{2}=m_{1}^{2}+\beta_{1} \eta_{1}-\varepsilon \beta_{2}$.

Decoupling (20) and (21), we obtain

$$
\left(\nabla^{2}+\chi_{1}^{2}\right)\left(\nabla^{2}+\chi_{2}^{2}\right)\left(\xi, T^{*}\right)=0
$$

where $\beta_{4}=\frac{\beta_{1}}{l}, \chi_{1}^{2}$ and $\chi_{2}^{2}$ are the roots with positive real parts of the biquadratic equation:

$$
\chi^{4}+\left(m_{1}^{2}+\beta_{4}^{2}-\eta_{1} \eta_{2}\right) \chi^{2}+m_{1}^{2} \beta_{4}^{2}=0
$$

Assuming the regularity conditions for $\xi$ and $T^{*}$ the solutions of (23) are obtained in terms of the spherical Hankel function, (23) representing an ordinary differential equation with variable coefficients of order four; from this equation we can determine the components of the displacement $U$ and the temperature $T$, and finally determine the components of the stress. We have

$$
\begin{aligned}
& \xi=K_{1} h_{0}^{(2)}\left(\chi_{1} r\right)+K_{2} h_{0}^{(2)}\left(\chi_{2} r\right), \\
& T^{*}=K_{1} h_{0}^{(2)}\left(\chi_{1} r\right)+K_{2} h_{0}^{(2)}\left(\chi_{2} r\right),
\end{aligned}
$$

where $K_{1}$ and $K_{2}$ are arbitrary constants and $h_{0}^{(2)}$ is the Hankel function of order zero and of the second kind. From (14), (18), (16), and (25a)-(25b), the solution for the displacement, temperature and the radial and hoop stresses are found to have the forms

$$
\begin{aligned}
& U=\left\{A h_{1}^{(2)}\left(\chi_{1} r\right)+B h_{1}^{(2)}\left(\chi_{2} r\right)\right\} e^{i \omega t^{\prime}}, \\
& T=\left\{K_{1} h_{0}^{(2)}\left(\chi_{1} r\right)+K_{2} h_{0}^{(2)}\left(\chi_{2} r\right)\right\} e^{i \omega t^{\prime}},
\end{aligned}
$$




$$
\begin{aligned}
\sigma_{r r}= & \left\{Q_{1} h_{0}^{(2)}\left(\chi_{1} r\right)+\frac{Q_{2}}{r} h_{1}^{(2)}\left(\chi_{1} r\right)\right\} A e^{i \omega t^{\prime}} \\
& +\left\{Q_{3} h_{0}^{(2)}\left(\chi_{2} r\right)+\frac{Q_{2}}{r} h_{1}^{(2)}\left(\chi_{2} r\right)\right\} B e^{i \omega t^{\prime}}, \\
\sigma_{\theta \theta}= & \left\{Q_{4} h_{0}^{(2)}\left(\chi_{1} r\right)+\frac{Q_{5}}{r} h_{1}^{(2)}\left(\chi_{1} r\right)\right\} A e^{i \omega t^{\prime}} \\
& +\left\{Q_{6} h_{0}^{(2)}\left(\chi_{2} r\right)+\frac{Q_{5}}{r} h_{1}^{(2)}\left(\chi_{2} r\right)\right\} B e^{i \omega t^{\prime}} .
\end{aligned}
$$

\section{Boundary conditions}

Let us consider the corresponding boundary conditions,

$$
\begin{aligned}
& u(1, t)=0, \quad r=1, \\
& \sigma_{r r}+\sigma_{r r}^{*}=-\sigma_{0} e^{i \omega t}, \quad r=1,
\end{aligned}
$$

where $\sigma_{0}$ is a constant; we get

$$
\begin{aligned}
z_{i}= & \frac{\chi_{i}^{2}-m_{1}^{2}}{t^{\prime} \chi_{i}}, \quad K_{i}=z_{i} A_{i}, \quad i=1,2, \\
A_{1}= & -\frac{\sigma_{0}^{\prime} h_{0}^{(2)}\left(\chi_{2}\right)}{d_{1}}, \quad A_{2}=\frac{\sigma_{0}^{\prime} z_{1} h_{0}^{(2)}\left(\chi_{1}\right)}{d_{1}}, \quad \sigma_{0}^{\prime}=\frac{\sigma_{0}}{\gamma T_{0}}, \\
d_{1}= & z_{2} h_{0}^{(2)}\left(\chi_{2}\right)\left\{Q_{1} h_{0}^{(2)}\left(\chi_{1}\right)+Q_{2} h_{1}^{(2)}\left(\chi_{1}\right)\right\} \\
& -z_{1} h_{0}^{(2)}\left(\chi_{1}\right)\left\{Q_{3} h_{0}^{(2)}\left(\chi_{2}\right)+Q_{2} h_{1}^{(2)}\left(\chi_{2}\right)\right\}, \\
Q_{1}= & \left(1+i \omega \tau_{0}^{\prime}+\beta_{4}^{2}\right) \chi_{1}-\left(1+i \omega \tau_{2}^{\prime}\right) z_{1}, \quad Q_{2}=\left(1+i \omega \tau_{0}^{\prime}+\beta_{4}^{2}\right)\left(2 \lambda_{e}-2\right), \\
Q_{3}= & \left(1+i \omega \tau_{0}^{\prime}+\beta_{4}^{2}\right) \chi_{2}-\left(1+i \omega \tau_{2}^{\prime}\right) z_{2}, \quad Q_{4}=\left(1+i \omega \tau_{0}^{\prime}+\beta_{4}^{2}\right) \chi_{1}-\left(1+i \omega \tau_{2}^{\prime}\right) z_{1}, \\
Q_{5}= & \left(1+i \omega \tau_{0}^{\prime}+\beta_{4}^{2}\right)\left(1-\lambda_{e}\right), \quad Q_{6}=\lambda_{e}\left(1+i \omega \tau_{0}^{\prime}+\beta_{4}^{2}\right) \chi_{2}-\left(1+i \omega \tau_{2}^{\prime}\right) z_{2}, \\
\beta_{4}^{2}= & \frac{\mu_{e}^{0} H_{\varphi}^{2}}{\rho c^{2}}, \quad m_{1}^{2}=\frac{\lambda}{a^{2} c^{2}\left(1+i \omega \tau_{0}^{\prime}+\beta_{4}^{2}\right)}, \quad \lambda_{e}=\frac{\lambda+2 \mu+P}{\lambda+} .
\end{aligned}
$$

This is the solution of the current problem for the case of a non-homogeneous isotropic viscoelastic unbounded body with spherical cavity without the effect of a magnetic field; it coincides with one previously published.

\section{Discussion and numerical results}

The results presented in this paper should prove useful for researchers in material science, designers of new materials, low-temperature physicists as well as for those working on the development of magneto-thermo-viscoelastic theory. The copper material was used chosen for purposes of numerical evaluations. The constants of the problem are given [24]. The numerical technique outlined above was used to obtain the temperature, radial displacement, radial stress and hoop stress inside the sphere. These distributions are shown in Figures 1-4, respectively. Important phenomena are observed in all computations: It was found that for large values of time the coupled and the generalized give close results. The case is quite different when we consider a small value of time. The coupled theory 

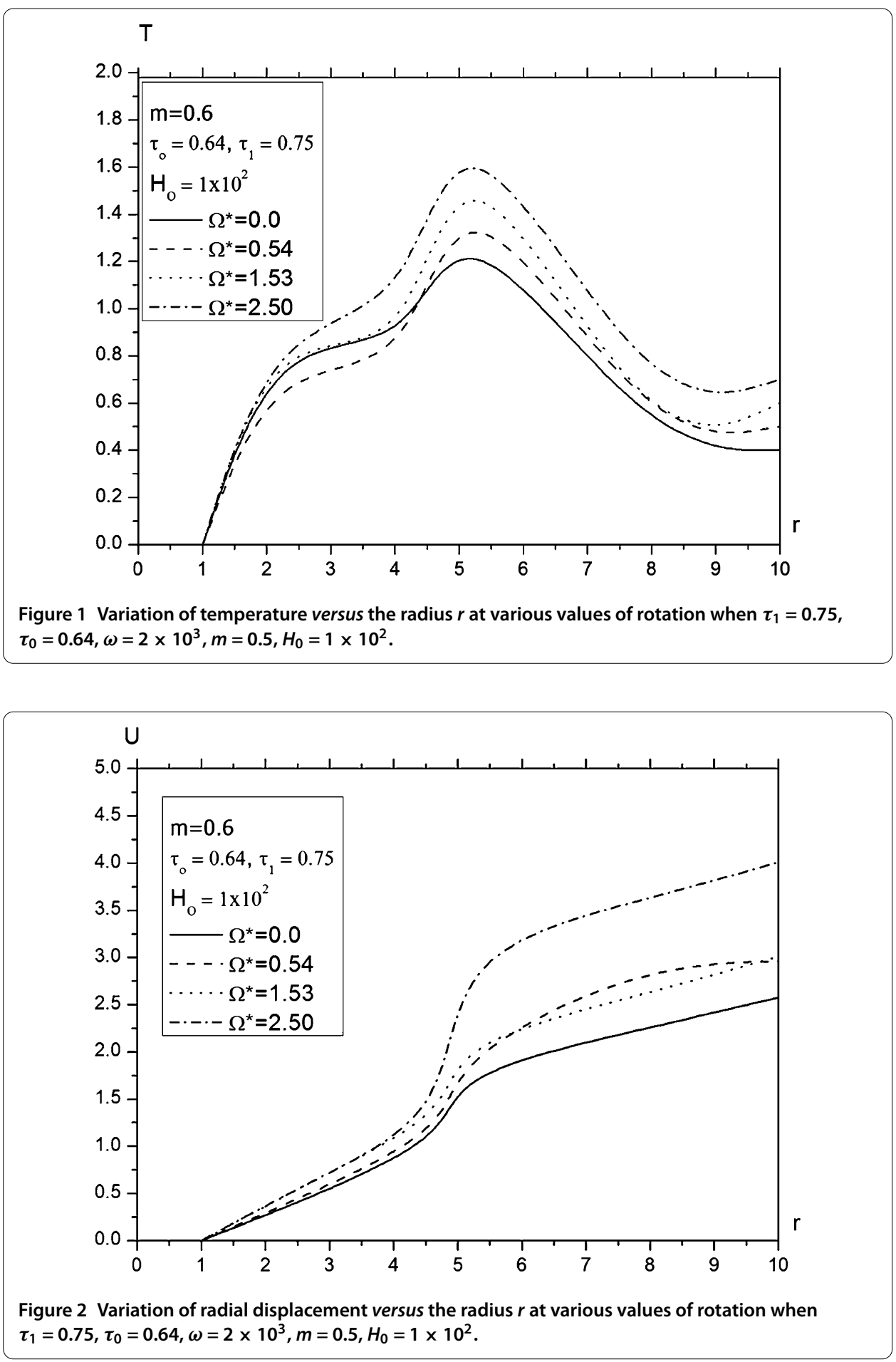

predicts infinite speeds of wave propagation. This is evident from the fact that the obtained solutions are not identically zero for any values of time but fade gradually to very small values at points for removed from the surface. The solutions obtained in the context of GL theory, however, exhibit the behavior of finite speeds of wave propagation. The computations were carried out for a thermal relaxation time of $\tau_{1}=0.75$, a magnetic field of $H_{0}=1 \times 10^{2}$, a mechanical relaxation time of $\tau_{0}=0.64$, and a frequency of $\omega=2 \times 10^{2}$. 


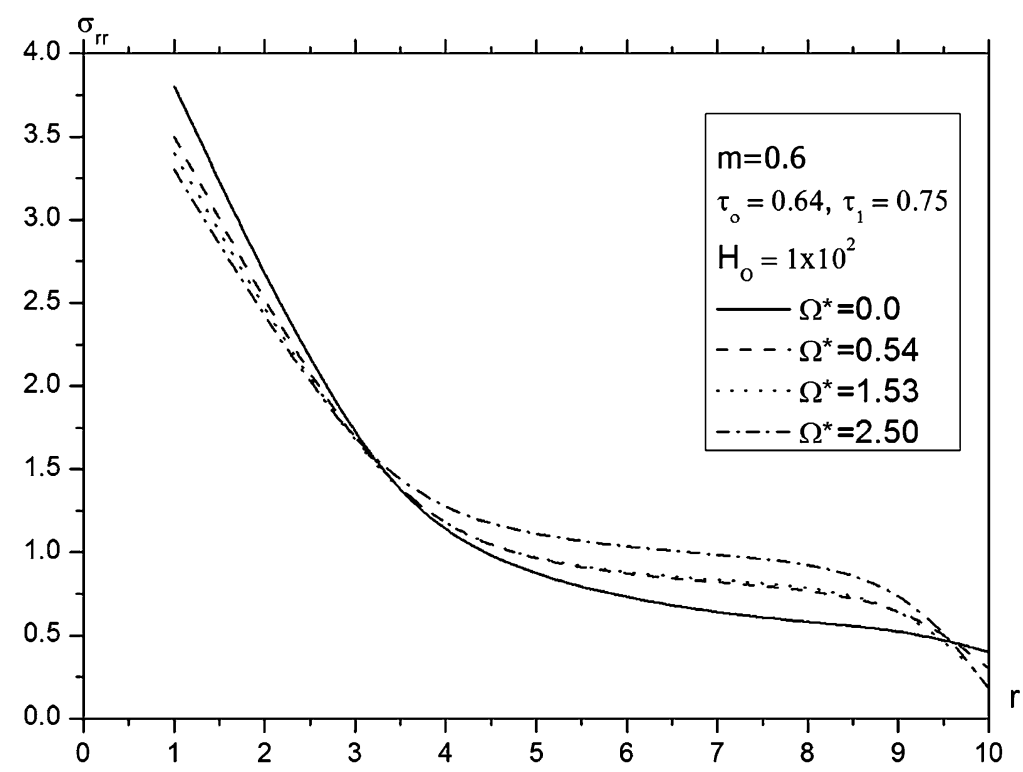

Figure 3 Variation of radial stress versus the radius $r$ at various values of rotation when $\tau_{1}=0.75$, $\tau_{0}=0.64, \omega=2 \times 10^{3}, m=0.5, H_{0}=1 \times 10^{2}$.

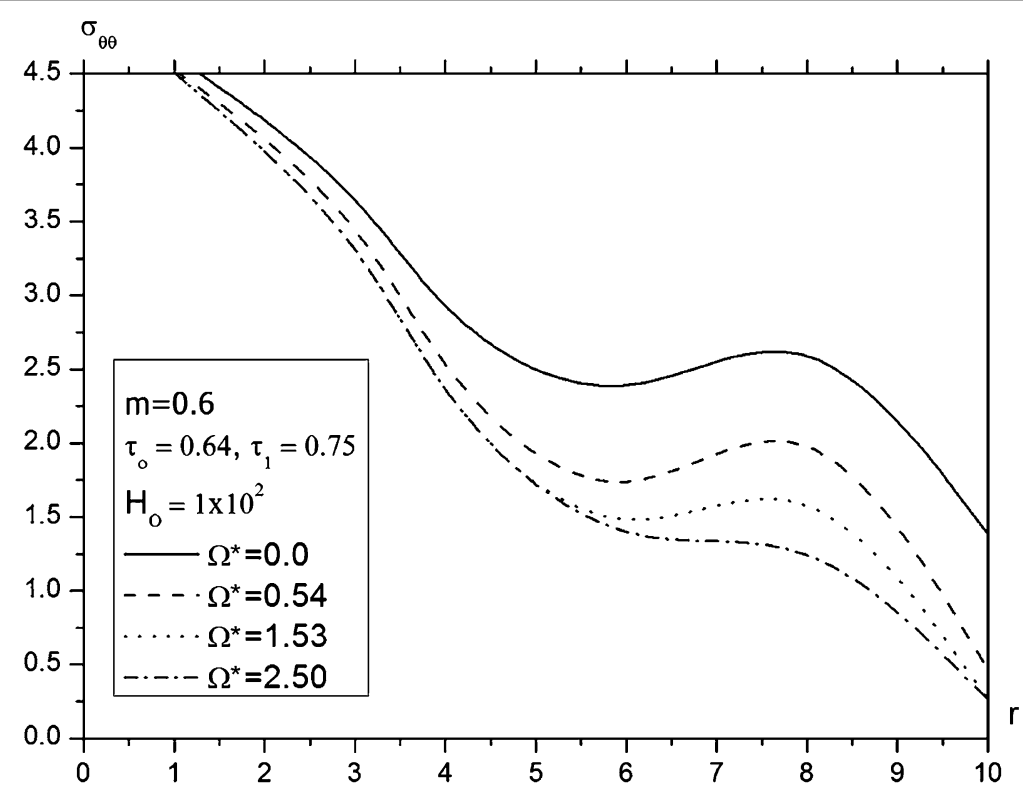

Figure 4 Variation of hoop stress versus the radius $r$ at various values of rotation when $\tau_{1}=0.75$, $\tau_{0}=0.64, \omega=2 \times 10^{3}, m=0.5, H_{0}=1 \times 10^{2}$.

For the sake of brevity some computational results are not presented here. Figures 1-4 show the solution corresponding to the use of the non-homogeneous material $(m=0.65)$. Figure 1 shows the temperature distribution and the solution corresponding to the effect of rotation. Figure 2 shows the radial displacement in a generalized thermo-elastic nonhomogeneous medium subjected to rotation, thermal relaxation, and a magnetic field. The figures indicate that the medium along the radius $r$ undergoes expansion deformation because of these effects. The radial displacement increases with increasing rotation 
and it increases with increasing radius $r$. Figure 3 shows the radial stress in a generalized thermo-elastic non-homogeneous medium subjected to rotation, thermal relaxation, and a magnetic field; in Figure 3 the radial stress decreases with increasing rotation when we have a small value of the radius $r$ of less than 2. Also, Figure 3 represents the solution corresponding to the use of the effect of the magnetic field $H_{0}$. Figure 4 shows the hoop stress in a generalized thermo-elastic non-homogeneous medium subjected to rotation, thermal relaxation, and a magnetic field. From this figure, the hoop stress decreases with increasing radius $r$; Figure 4 represents the solution corresponding to the use of the effect of rotation; also Figure 4 represents the hoop stress corresponding to the use of the effect of the magnetic field $H_{0}$. It was found that near the surface cavity where the boundary conditions dominate the coupled and the generalized theories give very close results. Inside the sphere, the solution is markedly different. This is due to the fact that thermal waves in the coupled theory the traveled distance is not identically zero (though it may be very small) for any small of time. By comparing with results in [24] it was found that $U$ has the same behavior in both media. But the values of $u$ in a generalized thermo-elastic medium are larger in comparison with those in a thermo-elastic medium. The same remark can be made for $\sigma_{r r}$ by comparing the figures. This is due to the influence of relaxation time, magnetic field, and frequency. Extensive literature on the topic is now available and we can only mention a few recent interesting investigations in [25-34].

\section{Conclusions}

The elasto-dynamic equations for the generalized thermo-viscoelastic theory under the effect of the non-homogeneous material, rotation, relaxation, and magnetic field have a complicated nature. The method used in this study provides a quite successful approach in dealing with such problems. The displacement, temperature, and stress components have been obtained in analytical form. This approach gives an exact solution in the Hankel transform domain that appears in the governing equations of the problem considered. Numerical results are calculated and discussed and illustrated graphically.

Competing interests

The authors declare that they have no competing interests.

Authors' contributions

All authors, KSA, SRM and EOA contributed to each part of this work equally and read and approved the final version of the manuscript.

Author details

${ }^{1}$ Mathematics Department, Faculty of Science, King Abdulaziz University, P.O. Box 80203, Jeddah, 21589, Saudi Arabia.

${ }^{2}$ Mathematics Department, Faculty of Science, Sohag University, Sohag, 82524, Egypt.

\section{Acknowledgements}

This article (project) was funded by the Deanship of Scientific Research (DSR), King Abdulaziz University, Jeddah, under grant No. (130-094-D1434). The authors, therefore, acknowledge with thanks DSR technical and financial support.

Received: 16 April 2014 Accepted: 25 June 2014 Published online: 24 September 2014

References

1. Yang, GC, Xu, YZ, Dang, LF: On the shear stress function and the critical value of the Blasius problem. J. Inequal. Appl. 2012, 208 (2012)

2. Ma, Z: Exponential stability and global attractors for a thermoelastic Bresse system. Adv. Differ. Equ. 2010, Article ID 748789 (2010)

3. Marin, M, Agarwal, RP, Mahmoud, SR: Modeling a microstretch thermoelastic body with two temperatures. Abstr. Appl. Anal. 2013, Article ID 583464 (2013). doi:10.1155/2013/583464

4. Emamizadeh, B: Applications of a weighted symmetrization inequality to elastic membranes and plates. J. Inequal. Appl. 2010, Article ID 808693 (2010). doi:10.1155/2010/808693 
5. Mahmoud, SR, Abd-Alla, AM, Al-Shehri, NA: Effect of the rotation on plane vibrations in a transversely isotropic infinite hollow cylinder. Int. J. Mod. Phys. B 25(26), 3513-3528 (2011)

6. Mahmoud, SR, Abd-Alla, AM, Matooka, BR: Effect of the rotation on wave motion through cylindrical bore in a micropolar porous cubic crystal. Int. J. Mod. Phys. B 25, 2713-2728 (2011)

7. Abd-Alla, AM, Yahya, GA, Mahmoud, SR: Effect of magnetic field and non-homogeneity on the radial vibrations in hollow rotating elastic cylinder. Meccanica 48(3), 555-566 (2013)

8. Abd-Alla, AM, Mahmoud, SR, Al-Shehri, NA: Effect of the rotation on a non-homogeneous infinite cylinder of orthotropic material. Appl. Math. Comput. 217, 8914-8922 (2011)

9. Abd-Alla, AM, Yahya, GA, Mahmoud, SR: Radial vibrations in a non-homogeneous orthotropic elastic hollow sphere subjected to rotation. J. Comput. Theor. Nanosci. 10(2), 455-463 (2013)

10. Abd-Alla, AM, Mahmoud, SR: Magneto-thermoelastic problem in rotating non-homogeneous orthotropic hollow cylinder under the hyperbolic heat conduction model. Meccanica 45, 451-462 (2010)

11. Mahmoud, SR: Analytical solution for electrostatic potential on wave propagation modeling in human long wet bones. J. Comput. Theor. Nanosci. 11(2), 454-463 (2014)

12. Mahmoud, SR: Influence of rotation and generalized magneto-thermoelastic on Rayleigh waves in a granular medium under effect of initial stress and gravity field. Meccanica 47(7), 1561-1579 (2012)

13. Abd-Alla, AM, Mahmoud, SR: Analytical solution of wave propagation in non-homogeneous orthotropic rotating elastic media. J. Mech. Sci. Technol. 26(3), 917-926 (2012)

14. Abd-Alla, AM, Mahmoud, SR, Abo-Dahab, SM, Helmi, MIR: Propagation of S-wave in a non-homogeneous anisotropic incompressible and initially stressed medium under influence of gravity field. Appl. Math. Comput. 217(9), 4321-4332 (2011)

15. Abd-Alla, AM, Abo-Dahab, SM, Mahmoud, SR, Hammad, HA: On generalized magneto-thermoelastic Rayleigh waves in a granular medium under influence of gravity field and initial stress. J. Vib. Control 17, 115-128 (2011)

16. Abd-Alla, AM, Mahmoud, SR, Abo-Dahab, SM: On problem of transient coupled thermoelasticity of an annular fin Meccanica 47(5), 1295-1306 (2012)

17. Abd-Alla, AM, Mahmoud, SR, Abo-Dahab, SM: Wave propagation modeling in cylindrical human long wet bones with cavity. Meccanica 46(6), 1413-1428 (2011)

18. Abd-Alla, AM, Mahmoud, SR: On problem of radial vibrations in non-homogeneity isotropic cylinder under influence of initial stress and magnetic field. J. Vib. Control 19(9), 1283-1293 (2013)

19. Mukhopadhyay, S: Effects of thermal relaxations on thermo-visco-elastic interactions in an unbounded body with a spherical cavity subjected to a periodic loading on the boundary. J. Therm. Stresses 23, 675-684 (2000)

20. Roychoudhuri, SK, Mukhopadhyay, S: Effect of rotation and relaxation times on plane waves in generalized thermo-viscoelasticity. Int. J. Math. Math. Sci. 23(7), 497-505 (2000)

21. Erbay, HA, Erbay, S, Dost, S: Thermally induced vibrations in a generalized thermoelastic solid with a cavity. J. Therm. Stresses 14, 161-171 (1991)

22. Li, J, Qi, J: Spectral problems for fractional differential equations from nonlocal continuum mechanics. Adv. Differ. Equ. 2014, 85 (2014)

23. Mahmoud, SR: Analytical solution for free vibrations of elastodynamic orthotropic hollow sphere under the influence of rotation. J. Comput. Theor. Nanosci. 11, 137-146 (2014)

24. Roychoudhuri, SK, Banerjee, S: Magneto-thermoelastic interactions in an infinite viscoelastic cylinder of temperature rate dependent material subjected to a periodic loading. Int. J. Eng. Sci. 36(5/6), 635-643 (1998)

25. Abd-Alla, AM, Abo-Dahab, SM, Mahmoud, SR, Al-Thamalia, TA: Influence of the rotation and gravity field on Stonely waves in a non-homogeneous orthotropic elastic medium. J. Comput. Theor. Nanosci. 10(2), 297-305 (2013)

26. Mahmoud, SR: On problem of shear waves in a magneto-elastic half-space of initially stressed a non-homogeneous anisotropic material under influence of rotation. Int. J. Mech. Sci. (2013). doi:10.1016/j.jjmecsci.2013.10.004

27. Marin, M: The Lagrange identity method in thermoelasticity of bodies with microstructure. Int. J. Eng. Sci. 32(8), 1229-1240 (1994)

28. Mahmoud, SR: Effect of non-homogeneity and rotation on an infinite generalized thermoelastic diffusion medium with a spherical cavity subject to magnetic field and initial stress. Abstr. Appl. Anal. 2013, Article ID 284646 (2013)

29. Marin, M: A partition of energy in thermoelasticity of microstretch bodies. Nonlinear Anal., Real World Appl. 11(4), 2436-2447 (2010)

30. Bessaim, A, Houari, MSA, Tounsi, A, Mahmoud, SR, Adda Bedia, EA: A new higher-order shear and normal deformation theory for the static and free vibration analysis of sandwich plates with functionally graded isotropic face sheets. J. Sandw. Struct. Mater. (2013). doi:10.1177/1099636213498888

31. Mahmoud, SR, Marin, M, Ali, Sl, Al-Basyouni, KS: On free vibrations of elastodynamic problem in rotating non-homogeneous orthotropic hollow sphere. Math. Probl. Eng. 2013, Article ID 250567 (2013)

32. Marin, M, Marinescu, C: Thermoelasticity of initially stressed bodies, asymptotic equipartition of energies. Int. J. Eng. Sci. 36(1), 73-86 (1998)

33. Ezzat, MA, Atef, HM: Magneto-thermo-viscoelastic material with a spherical cavity. J. Civ. Eng. Constr. Technol. 2(1), 6-16 (2011)

34. Marin, M, Agarwal, RP, Mahmoud, SR: Nonsimple material problems addressed by the Lagrange's identity. Bound. Value Probl. 2013, 135 (2013). doi:10.1186/1687-2770-2013-135

doi:10.1186/s13661-014-0166-7

Cite this article as: Al-Basyouni et al.: Effect of rotation, magnetic field and a periodic loading on radial vibrations thermo-viscoelastic non-homogeneous media. Boundary Value Problems 2014 2014:166. 